\title{
LncRNA BX357664 inhibits cell proliferation and invasion and promotes cell apoptosis in human colorectal cancer cells
}

\author{
FENGJUN LIU ${ }^{1 *}$, XINSHENG WANG ${ }^{1,2^{*}}$, HAIYAN LIU ${ }^{3}$, YANG WANG $^{4}$, \\ XIAOQIAN LIU ${ }^{5}$, XIAOCHEN HAO ${ }^{6}$ and HONGGUANG $\mathrm{LI}^{7}$
}

\begin{abstract}
${ }^{1}$ Department of General Surgery, Shandong University Qilu Hospital, Jinan, Shandong 250012; ${ }^{2}$ Department of General Surgery, Anqiu People's Hospital; ${ }^{3}$ Department of Rehabilitation, Anqiu Maternal and Child Health Care Hospital, Anqiu, Shandong 262100; ${ }^{4}$ Department of General Surgery, Weifang People's Hospital, Weifang, Shandong 261000;

${ }^{5}$ Department of Obstetrics, Anqiu Maternal and Child Health Care Hospital; ${ }^{6}$ Department of Internal Medicine Cardiovascular, Anqiu People's Hospital; ${ }^{7}$ Department of Urology Surgery, Anqiu People's Hospital, Anqiu, Shandong 262100, P.R. China
\end{abstract}

Received May 17, 2017; Accepted November 21, 2017

DOI: $10.3892 / \mathrm{ol} .2018 .8435$

\begin{abstract}
Colorectal cancer represents a great burden for patients worldwide. Long noncoding RNA BX357664 is an RNA that was identified by microarray technique in renal cell carcinoma. The function of BX357664 in solid tumors remains largely unknown. The present study aimed to investigate the expression profile and functional role of BX357664 in human colorectal cancer progression. The transcription levels of BX357664 were initially examined in vivo and in vitro. An overexpression plasmid was constructed in order to examine the effects of BX357664 overexpression on cell proliferation, apoptosis, migration and invasion. The results demonstrated that BX357664 was significantly downregulated in clinical colorectal cancer tissues and cell lines. Overexpression of BX357664 decreased cell proliferation rates and cell colony formation capacities in HCT116 and HT-29 cells. Following BX357664 overexpression, HCT116 and HT-29 cells exhibited reduced migration and invasion capacities. Would closure was also blunted by $>50 \%$ following overexpression of BX357664 in HCT-116 and HT-29 cells. In addition, the cell cycle regulators Cyclin B1, CDC25C and Cyclin D1 as well as the mesenchymal marker N-cadherin were downregulated, whereas the epithelial marker E-cadherin was upregulated by BX357664 overexpression. Finally, HCT116 and HT-29 cell apoptosis was induced and activities of caspase- 3 and caspase-9 increased significantly following BX357664 overexpression. The present data suggested that BX257664 negatively regulated cell proliferation
\end{abstract}

Correspondence to: Dr Fengjun Liu, Department of General Surgery, Shandong University Qilu Hospital, 107 West Cultural Road, Jinan, Shandong 250012, P.R. China

E-mail: fj1234liu@sina.com

*Contributed equally

Key words: colorectal cancer, long noncoding RNA BX375664, proliferation, metastasis, apoptosis and metastasis and promoted cell apoptosis in colorectal cancer. These observations provided novel evidence that BX357664 might serve as a tumor suppressor and a potential therapeutic target in the treatment of colorectal cancer in the clinic.

\section{Introduction}

Colorectal cancer (CRC) is one of the most common causes of cancer-related deaths worldwide, with high mortality and morbidity in both genders (1-3). Current therapeutic strategies against CRC consist of curative surgery and postoperative adjuvant chemotherapy. Despite the slow and steady improvement in the overall 5-year survival rate during the past decades $(3,4)$, prognosis of patients with CRC remains poor due to post-surgical recurrence and fatal distant metastasis. According to a recent statistic, the 5-year relative survival ranges from $>90 \%$ for patients with stage I disease to slightly $>10 \%$ in patients with stage IV disease (5). Therefore, it is a great urgency to search for novel biomarkers, suitable intervention strategies in order to improve patient outcomes.

With the rapid development of genome and transcriptome sequencing technologies and implementation of genomics consortiums, such as ENCODE and FANTOM, the classic view of the transcriptome landscape and its mRNA-centric paradigm for transcript annotation has undergone a fundamental change (6). Current knowledge has well recognized that the vast majority of genome serves as the template for the transcription of noncoding RNAs. For example, small noncoding RNAs, particularly microRNAs, have been extensively investigated for several decades, and their biological functions in various cancers have been uncovered (7). However, as the fundamental elements of noncoding RNAs, long noncoding RNAs (lncRNAs) has not received a wide recognition and the function of lncRNAs in numerous cancers still remains to be elucidated.

LncRNAs are defined as a class of RNAs with length $>200$ nucleotides. Ever since its discovery, lncRNAs have been identified as key regulators of various biological processes, including cell proliferation, differentiation, apoptosis, migration, and invasion (8). The lncRNA UCC, for instance, has 
recently been identified to promote CRC cell growth and invasion by sponging miR-143 (9).

LncRNA BX357664 is a novel lncRNA that has been identified from the analysis of normalized microarray data and further quantitative polymerase chain reaction (qPCR) analysis. BX357664 might be a potential biomarker involved in renal cell carcinoma (10). Furthermore, it was demonstrated that BX357664 regulates cell proliferation and epithelial-to-mesenchymal transition (EMT) via inhibition of transforming growth factor (TGF)- $\beta 1 / \mathrm{p} 38 /$ heat shock protein (HSP) 27 signaling in renal cell carcinoma (11). These data implicated that BX357664 might have critical roles in human tumorigenesis. However, the role of BX357664 in other types of solid tumors remains largely unknown.

The present study aimed to investigate the role of BX357664 in human CRC. To this end, the expression levels of BX357664 in human CRC tissues and in CRC cell lines were examined. Expression of BX357664 was then modulated in order to assess the functional changes of CRC cells in response to BX357664 alteration. Cell survival was also examined. The present data imply that BX357664 may be a critical tumor suppressor that might serve as a drug target in the treatment of $\mathrm{CRC}$ in the clinic.

\section{Materials and methods}

Human samples. Colorectal cancer tissues from 80 patients (age range, 40-80 years; mean age, 58 years; male:female ratio, 47:33), admitted to the Department of General Surgery, Qilu Hospital of Shandong University (Jinan, China) between March 2016 and March 2017, were collected via surgical resection, frozen into liquid nitrogen immediately once dissected from patients, and stored at $-80^{\circ} \mathrm{C}$. Matched adjacent non-cancerous tissues were also obtained from each CRC patient. All patients expressed their full intentions to participate in the present study and written consent forms were obtained from each patient. The present study was approved by the Ethics Committee of the Anqiu People's Hospital (Weifang, China).

Cell culture and plasmid transfection. Control cells HIEC-6 were purchased from the American Type Tissue Collection (Manassas, VA, USA). CRC cell lines COLO 205, HCT116 and HT-29 were purchased from the Cell Bank of Chinese Academy of Sciences (Shanghai, China). All of the cell lines were cultured in DMEM (Gibco; Thermo Fisher Scientific, Inc., Waltham, MA, USA) supplemented with $10 \%$ fetal bovine serum (FBS; Gibco; Thermo Fisher Scientific, Inc.) as well as $1 \%$ antibiotics (penicillin/streptomycin). The cell lines were cultured in a $37^{\circ} \mathrm{C}$ incubator with $5 \% \mathrm{CO}_{2}$ and the culture media were replaced every other day, otherwise as stated. The full sequence of BX357664 was cloned from human genome extracted from normal human liver, and then cloned into pcDNA 3.1 vector (catalog no., 52535; Addgene, Inc., Cambridge, MA, USA) at the sites of the restriction enzymes EcoRI and XhoI. The correct sequence was confirmed by sequencing using the T7 promoter forward primer (Shenggong Biology Engineering Technology Service, Ltd., Shanghai, China). The transfections were performed with Lipofectamine 2000 (Invitrogen; Thermo Fisher Scientific, Inc.), according to the manufactures' instructions, using a dose of $2.5 \mu \mathrm{l}$ for
$1.5 \mu \mathrm{g}$ plasmid DNA. Six h following transfection, the culture medium was replaced with fresh DMEM.

RNA isolation and reverse transcription $(R T)-q P C R$. Total RNA from CRC patient tissues and cultured CRC cells were extracted with TRIzol reagent (Takara Biotechnology Co., Ltd., Dalian, China) in a dilution of $1 \mathrm{ml}$ for each well in a six-well plate. The RNA quality and quantity were determined using a Nanodrop 2000 spectrophotometer (Thermo Fisher Scientific, Inc.) at the absorbance of 260 and $280 \mathrm{~nm}$. Reverse transcription of first-strand cDNA was performed with PrimeScript RT Master Mix (Perfect Real Time; Takara Biotechnology Co., Ltd.), following the manufacturer's protocol. All PCR reactions were performed in an ABI PRISM 7900 Real-Time system (Thermo Fisher Scientific, Inc.) with the SYBR Premix Ex Taq kit (Takara Biotechnology Co., Ltd.). The thermocycling protocol was as follows: Initial denaturation at $95^{\circ} \mathrm{C}$ for $2 \mathrm{~min}$, followed by 35 repeats of the three-step cycling program consisting of $30 \mathrm{sec}$ at $95^{\circ} \mathrm{C}$ (denaturation), $1 \mathrm{~min}$ at $53^{\circ} \mathrm{C}$ (primer annealing) and $30 \mathrm{sec}$ at $72^{\circ} \mathrm{C}$ (elongation), followed by a final extension step for $10 \mathrm{~min}$ at $72^{\circ} \mathrm{C}$. The housekeeping gene GAPDH was included as an internal control. Primers were purchased from Shenggong Biology Engineering Technology Service, Ltd. (Shanghai, China) with the following sequences: BX357664, forward, 5'-GGCGTGGTTTTGATGGAGTG-3', and reverse, 5'-AGG CTGCAGAGTTGAGATCG-3'; GAPDH, forward, 5'-GTG GACATCCGCAAAGAC-3' and reverse, 5'-AAAGGGTGT AACGCAACTA-3'. All quantitative data were normalized to GAPDH using the $2^{-\Delta \Delta C q}$ method (12).

Colony formation assay. HCT116 and HT-29 cells were transfected with pcDNA 3.1 vector with or without BX357664 sequence in six-well plates with a density of 200 cells/well. After 2 weeks in a $37^{\circ} \mathrm{C}$ incubator, cell colonies that contained $>50$ cells were counted by staining with crystal violet $(0.5 \%$, $10 \mathrm{~min}$ ) and observation under a light microscope with a magnification of x200 (Nikon Corporation, Tokyo, Japan).

Cell cycle analysis. Prior to cell cycle analysis, HCT116 and HT-29 cells were transfected with empty vector plasmid or BX357664 plasmid for $48 \mathrm{~h}$. Next, cells were collected with low speed centrifugation $\left(840 \mathrm{x} \mathrm{g}, 5 \mathrm{~min}, 4^{\circ} \mathrm{C}\right)$ and fixed with pre-iced ethanol $(70 \%)$ for $10 \mathrm{~min}$ at $4^{\circ} \mathrm{C}$. The cells were washed and re-suspended in cold PBS and incubated at $37^{\circ} \mathrm{C}$ for $30 \mathrm{~min}$ with $10 \mathrm{mg} / \mathrm{ml}$ RNase and $1 \mathrm{mg} / \mathrm{ml}$ propidium iodide (PI; Sigma-Aldrich; Merck KGaA, Darmstadt, Germany). The \% of cells in each phase of the cell cycle was determined using the Cell Quest Pro acquisition software (BD Biosciences, Franklin Lakes, NJ, USA).

Cell viability determination. Both HCT116 and HT-29 cells were seeded in a 96-well plate at a concentration of 1,000 cells/well. Following incubation for $24 \mathrm{~h}$, cells were transfected with BX357664-expressing plasmid or control vector. Cell proliferation was examined in consecutive 5 days with a Cell Titer 96 AQueous Non-Radioactive Cell Proliferation kit (Promega Corporation, Madison, WI, USA), as per the manufacturers' protocol. The cell proliferative rate in each group was determined by measuring the absorbance 
at $490 \mathrm{~nm}$ using a microplate reader (Tecan, Männedorf, Switzerland). For data presentation, absorbance for control cells at the first day was set as 1 and used for normalization. Absorbance values for other groups were then normalized to that of control at the first day. Therefore, cell viability ratio was calculated as the absorbance in others groups relative to that in control group at the first day.

Transwell assay. For cell migration assays, HCT116 and HT-29 cells were transfected with corresponding plasmid for $48 \mathrm{~h}$ and then trypsinized, collected by low-speed centrifugation $\left(840 \mathrm{x} \mathrm{g}, 4^{\circ} \mathrm{C}, 5 \mathrm{~min}\right)$ in serum-free medium. A total of $1 \times 10^{4}$ cells $(200 \mu \mathrm{l})$ were seeded into the upper chamber of a Corning Transwell chamber ( $8 \mu \mathrm{m}$ pore). The lower chamber was filled with $600 \mu 1$ medium containing 10\% FBS. Afterwards, the plate was incubated at $37^{\circ} \mathrm{C}$ and the cells are allowed to migrate for $24 \mathrm{~h}$. Afterwards, the membrane was fixed with pre-cooled methanol and stained with crystal violet (1\%) for $5 \mathrm{~min}$ at room temperature. Cell migration was assessed by counting the cells that had migrated through the membrane. Five random fields were selected and images were captured under a Nikon light microscope (Nikon Corporation, Tokyo, Japan) at a magnification of x100. For cell invasion assays, the membrane was pre-coated with Matrigel (Corning Incorporated, Corning, NY, USA) for $6 \mathrm{~h}$ in $37^{\circ} \mathrm{C}$ incubator.

Wound-healing assay. HCT116 and HT-29 cells were transfected with BX357664-expressing or control plasmid, cultured in DMEM in a six-well culture plate at a density of $5 \times 10^{5}$ cells/well and allowed to grow to a confluence of $90 \%$ overnight. Next day, the culture medium was replaced with serum-free DMEM, and then two lines were scratched in the single cell layer with a $10 \mu l$ pipette tip and the cells were washed with PBS three times. Following incubation for $12 \mathrm{~h}$, images of the migrating cells were observed and captured using a Nikon light microscope at the cross of two straight lines.

Western blot analysis. Molecules included in the process of EMT and cell cycle progression were evaluated by western blot analysis. Briefly, HCT116 and HT-29 cells were transfected with BX357664-expressing plasmid for $48 \mathrm{~h}$, and then total proteins were prepared with lysis buffer (RIPA; Beyotime Institute of Biotechnology, Nantong, China) on ice and quantified using a Bio-Rad protein assay reagent (Thermo Fisher Scientific, Inc.). Equal amounts of protein $(50 \mu \mathrm{g})$ were separated with $10 \%$ SDS-PAGE and transferred to a nitrocellulose membrane (EMD Millipore, Billerica, MA, USA). The membrane was blocked for $1 \mathrm{~h}$ with $5 \%$ skimmed milk at room temperature and then incubated with primary antibodies overnight at $4^{\circ} \mathrm{C}$. The primary antibodies against Cyclin B1 (cat. no. sc-70898; 1:1,000), cell-division cycle (CDC) 25C (cat. no. sc-327; 1:1,000), Cyclin D1 (cat. no. sc-4074; 1:1,000), E-Cadherin (cat. no. sc-71009; 1:1,000), N-Cadherin (cat. no. sc-53488; 1:1,000) and GAPDH (cat. no. sc-32233; 1:1,000) were purchased from Santa Cruz Biotechnology, Inc. (Dallas, TX, USA). After washing with TBST for 4 times (8 min each), the membrane was incubated with secondary goat-anti-rabbit (cat. no. sc-2004) or goat-anti-mouse (cat. no. sc-2005) antibody (Santa Cruz Biotechnology, Inc.) for $1 \mathrm{~h}$ at $37^{\circ} \mathrm{C}$ with a dilution of 1:1,000. Finally, the proteins were quantified using ECL
Prime Western Blotting Detection reagent (GE Healthcare, Chicago, IL, USA) and an ImageQuant LAS 4000 Mini Biomolecular Imager (GE Healthcare).

Flow cytometric analysis of cell apoptosis. The annexin V/PI assay was performed as per the manufacturer's instructions (Invitrogen; Thermo Fisher Scientific, Inc.). Briefly, HCT116 and HT-29 cells were plated into 6-well plates and transfected with control or BX357664-expressing plasmid for 48 h. Afterwards, cells were washed with cold PBS, trypsinized, and re-suspended in $100 \mu \mathrm{l}$ of binding buffer with $2.5 \mu \mathrm{l}$ fluorescein isothiocyanate (FITC)-conjugated annexin V and $1 \mu \mathrm{l} \mathrm{PI}(100 \mu \mathrm{g} / \mathrm{ml})$. Afterwards, cells were incubated at room temperature for $15 \mathrm{~min}$ in the dark. A total of at least 10,000 cells were collected and analyzed by flow cytometry for both cell lines (Cell Quest Pro acquisition software, BD Biosciences, Franklin Lakes, NJ, USA).

Determination of caspase activities. The activities of caspase-3, caspase- 8 and caspase- 9 were determined by specific caspase activity kits (Beyotime Institute of Biotechnology), following the manufacturer's instructions. Briefly, cells were transfected with plasmids for $48 \mathrm{~h}$. Afterwards, cell lysates were collected by low speed centrifugation $\left(840 \mathrm{x} \mathrm{g}, 5 \mathrm{~min}, 4^{\circ} \mathrm{C}\right)$. An equal amount of $10 \mu \mathrm{l}$ proteins from each sample were added into 96-well plates and mixed with an aliquot of $80 \mu 1$ reaction buffer supplied with caspase substrates $(2 \mathrm{mM})$. Following incubation at $37^{\circ} \mathrm{C}$ for $4 \mathrm{~h}$, caspase activities were determined using a Tecan plate reader at an absorbance of $450 \mathrm{~nm}$.

Statistical analysis. All experiments were repeated at least three times in triplicate, showing reproducible results. All data were presented as the mean \pm standard deviation, except if otherwise stated. Student's t-test analysis was used for the comparison of numerical variables between two groups. One-way analysis of variance was used for comparisons of numerical variables among multiple groups, followed by the LSD post hoc test. Data were analyzed with GraphPad Prism 6 (GraphPad Software Inc., San Diego, CA, USA). P<0.05 was considered to indicate a statistically significant difference.

\section{Results}

BX357664 is downregulated in CRC patient tissues and cell lines. Initially, the expression profile of BX357664 was examined in clinical CRC tissues. To this end, a total of 80 cases with clinically diagnosed CRC were collected. Adjacent non-cancerous tissues were included in the analysis as a control. As illustrated in Fig. 1A, the transcription levels of BX357664 in cancerous tissues were significantly lower compared with adjacent normal tissues. The mean transcription level of BX357664 in cancerous tissue was only 25-50\% of that in the adjacent ones (Fig. 1A; P<0.001). Furthermore, compared with the normal intestinal epithelial control HIEC-6 cells, the expression of BX357664 in CRC cell lines (COLO205, HCT116 and HT-29) was significantly reduced (Fig. 1B). These data suggested that BX357664 was downregulated in CRC.

Overexpression of BX357664 inhibits cell growth and arrests cell cycle in HCT116 and HT-29 cells. Next, BX357664 was 

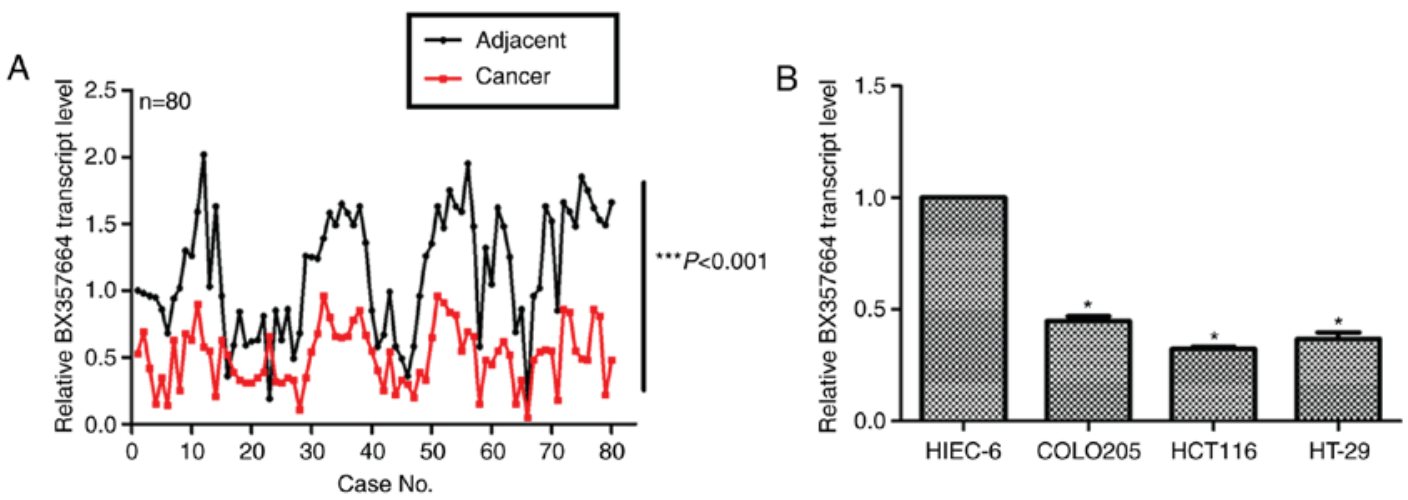

Figure 1. BX357664 is downregulated in colorectal cancer in vivo and in vitro. (A) RT-qPCR analysis of BX357664 transcript levels in 80 patient cases of $\mathrm{CRC}$ tissues and their matched adjacent non-cancerous tissues. The $\mathrm{x}$ axis represents the case serial number (nos. $1-80) .{ }^{* * *} \mathrm{P}<0.001 \mathrm{vs.}$ adjacent non-cancerous tissues. (B) RT-qPCR analysis of BX357664 transcript levels in HIEC-6 cells (control) and three CRC cell lines (COLO205, HCT116 and HT-29). "P<0.05 vs. HIEC-6. RT-qPCR, reverse transcription-quantitative polymerase chain reaction; CRC, colorectal cancer.

A
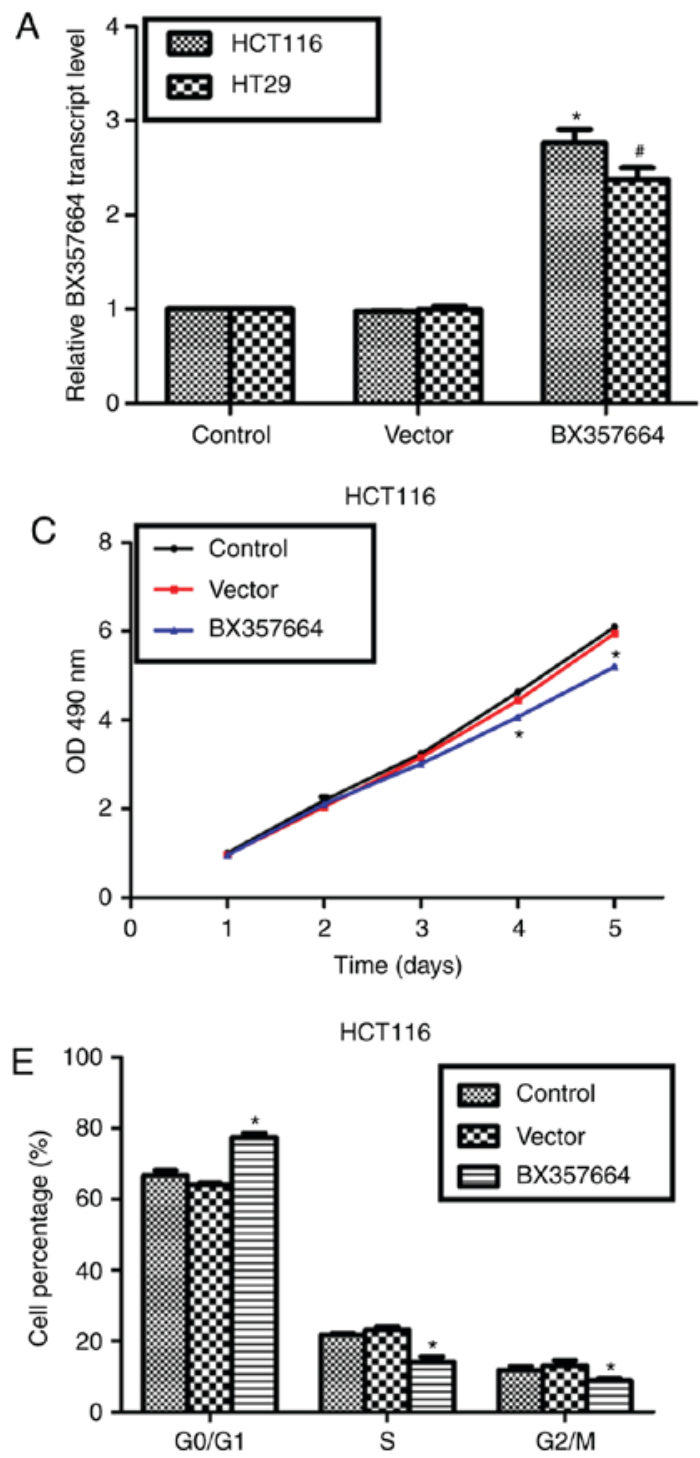
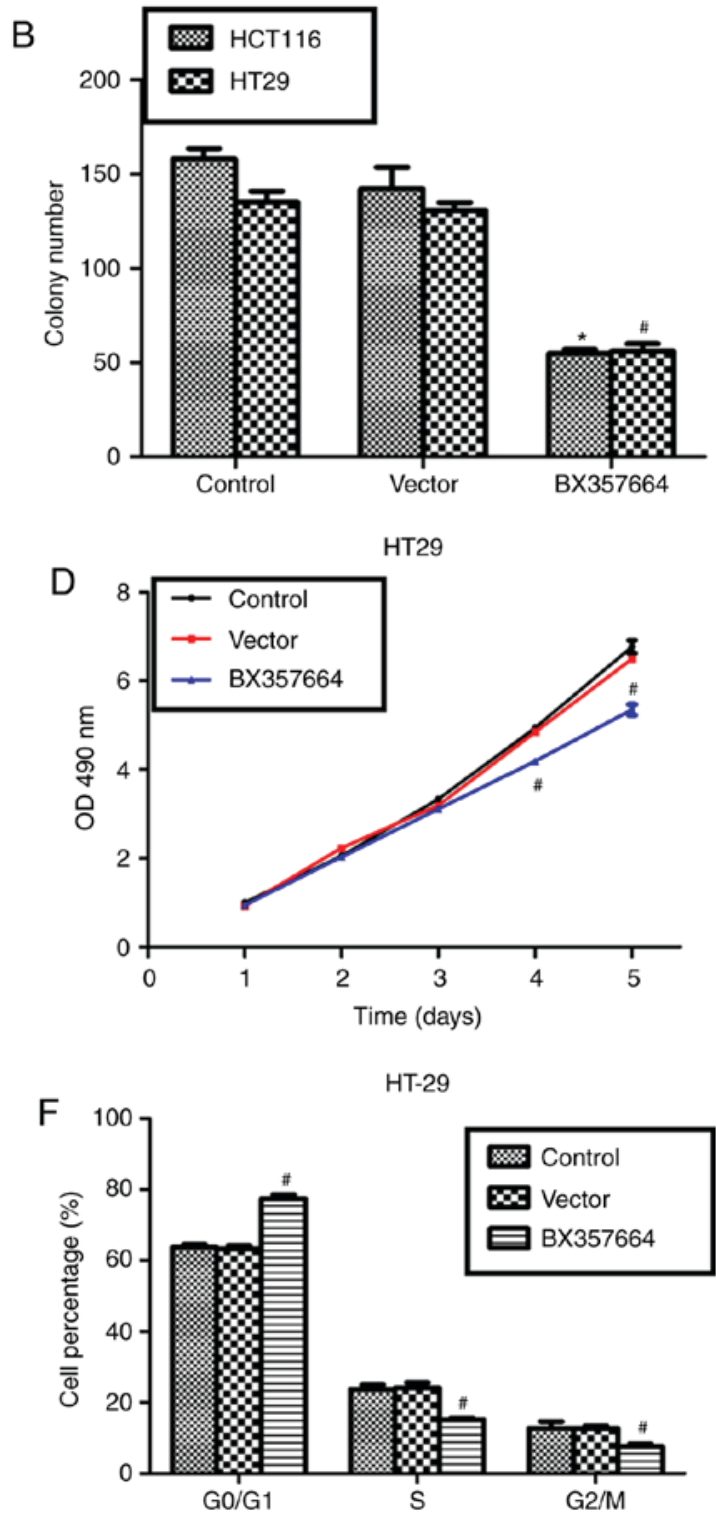

Figure 2. Overexpression of BX357664 inhibits cell growth and arrests cell cycle in HCT116 and HT-29 cells. (A) An overexpression plasmid was established to increase the expression of BX357664 in HCT116 cells and HT-29 cells. Reverse transcription-quantitative polymerase chain reaction analysis was performed to confirm the efficiency of the plasmid transfection. (B-D) Control non-transfected cells (control), cells transfected with empty vector (vector) or cells transfected with BX357664-expressing plasmid were subjected to colony formation assay or cell viability assays. (E, F) Control non-transfected cells (control), cells transfected with empty vector (vector) or cells transfected with BX357664-expressing plasmid were subjected to cell cycle analysis. Cell proportions (\%) in each phase of the cell cycle were determined. " $\mathrm{P}<0.05$ vs. HCT116 vector group; ${ }^{*} \mathrm{P}<0.05$ vs. HT 29 vector group. OD, optical density. 

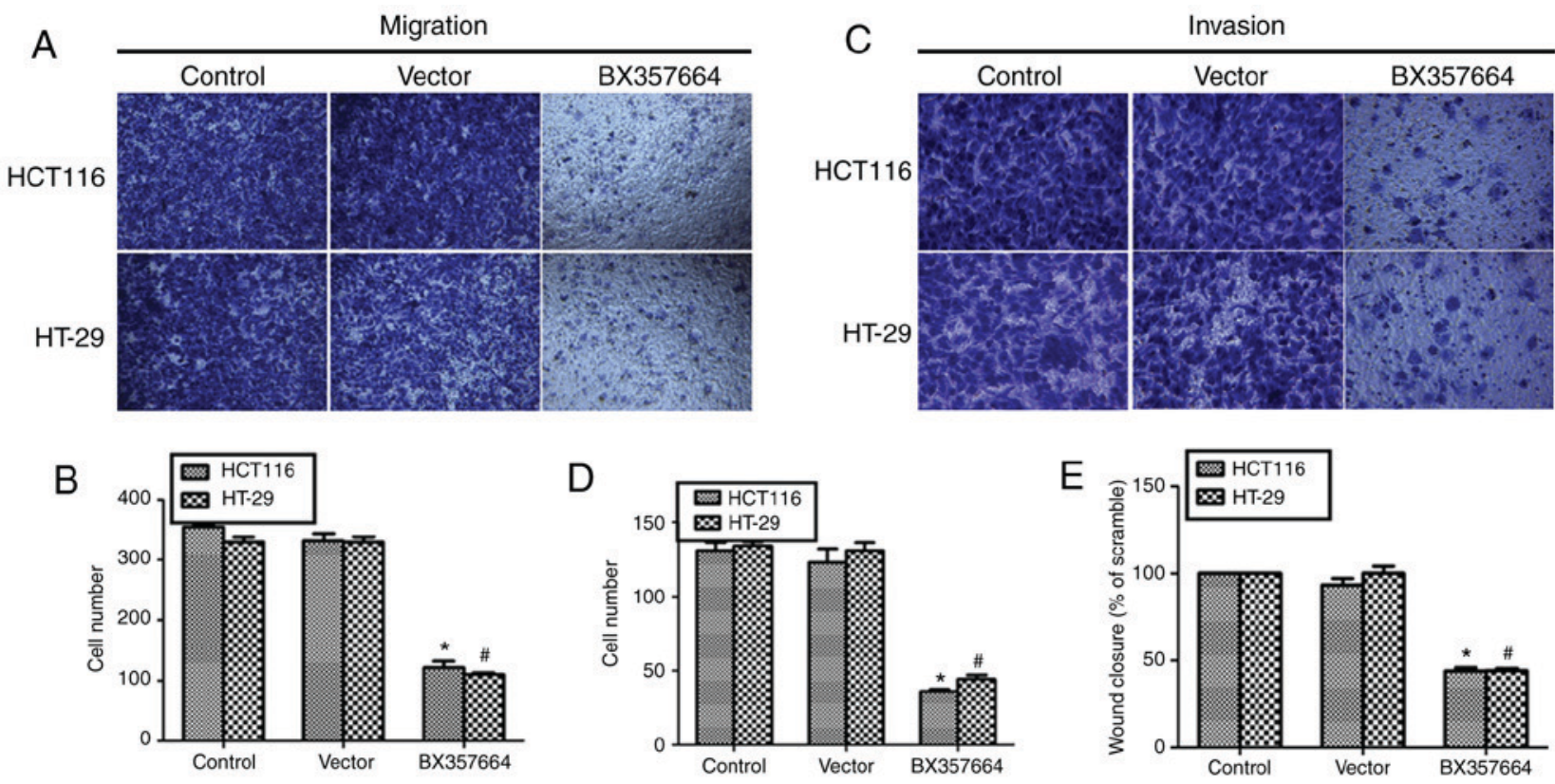

Figure 3. Overexpression of BX357664 inhibits cell migration and invasion in HCT116 and HT-29 cells. (A) Transwell migration assays were performed to assess cell migration capacities with or without BX357664 overexpression. Representative images from the transmigrated cells at the bottom of the chambers are shown (magnification, x100). (B) Cell numbers in the lower membrane of the migration transwell chambers were quantified from five random fields for each group. (C) Transwell invasion assays were performed to assess cell invasion capacities with or without BX357664 overexpression. Representative images from the invaded cells at the bottom of the chambers are shown (magnification, x100). (D) Quantification data from averaging five random fields for each group. (E) Wound healing assay was performed for HCT116 cells and HT-29 cells with or without BX357664 overexpression. Twelve h following the artificial scratch, would closure was photographed and the rate of wound closure was calculated and normalized to the vector-transfected group. * $<0.05$ vs. HCT116 vector group; ${ }^{\text {}} \mathrm{P}<0.05$ vs. HT 29 vector group.

overexpressed in HCT116 and HT-29 cells by transfection of an overexpression construct. Analysis of transcription levels of BX357664 revealed that the overexpression construct successfully elevated the expression of BX357664 in both cell lines by up to 2-3 fold, compared with the cells transfected with empty vector (Fig. 2A). With the aid of this overexpression system, colony formation and cell viability assays were performed. As presented in Fig. 2B, it was observed that transfection of a vector plasmid resulted in insignificant changes on cell capacities to form colonies. However, compared with an average of 150 colonies in control HCT116 cells and 135 in control HT-29 cells, BX357664-overexpressing cells exhibited a significantly reduced average of 50 colonies(Fig.2B).In the cell viability assay, it was observed that the number of BX357664-overexpressing cells was $\sim 70 \%$ of control HCT116 cells on the 5th day (Fig. 2C), while the number of BX357664-overexpressing cells was $\sim 65 \%$ of control HT-29 cells on the 5th day (Fig. 2D). In addition, cell cycle progression was assessed in control and BX357664-overexpressing cells. Following transfection of BX357664 into HCT116 cells, the \% of cells in the G0/G1 phase was significantly increased, whereas the $\%$ of cells in the $\mathrm{S}$ and G2/M phases was decreased accordingly (Fig. 2E). Likewise, in the HT-29 cells, cells were more accumulated in the G0/G1 phase following overexpression of BX357664, whereas control HT-29 cells were significantly more accumulated in the $\mathrm{S}$ and G2/M phases (Fig. 2F). These data suggested that overexpression of BX357664 led to cell growth inhibition and cell cycle arrest at the G0/G1 phase.

Overexpression of BX357664 inhibits cell migration and invasion in HCT116 and HT-29 cells. The effects of BX357664 overexpression on cell migration and invasion were next examined. In the transwell migration assay, there were visibly less cells migrated to the lower chamber observed in the BX357664-transfected group (Fig. 3A). Quantification of transmigrated cells demonstrated that almost 340 cells migrated to the lower chamber in the control groups, while only 100 cells with BX357664 overexpression were observed in the lower chamber, indicating a $70 \%$ decrease of migration capacity (Fig. 3B). Similarly, in the transwell invasion assay, cells that invaded into the lower chamber were visibly fewer in the BX357664-transfected HCT116 and HT-29 cells (Fig. 3C). In fact, an average of 126 control HCT116 cells were counted in the lower chamber while 34 cells were counted in the lower chamber of the BX357664-overexpressing HCT116 cells (Fig. 3D). For the HT-29 cell line, only 36 cells invaded through the Matrigel compared with $\sim 128$ cells in the control groups (Fig. 3D). Finally, in the wound healing assay, BX357664-overexpressing cells displayed significantly reduced capacities to recover the scratched wound, as evidenced by the lower rate of wound closure compared with the control groups (Fig. 3E). These findings suggested that overexpression of BX357664 inhibited cell migration and invasion in CRC cells.

Overexpression of BX357664 alters cell cycle regulator and EMT marker expression. In view of the above observations, the expression of key cell cycle regulators and EMT markers was examined in HCT116 and HT-29 cells with or without BX357664 overexpression. In HCT116 cells, overexpression of BX357664 decreased the protein levels of Cyclin B1, CDC25C and Cyclin D1 (Fig. 4A). In addition, expression 

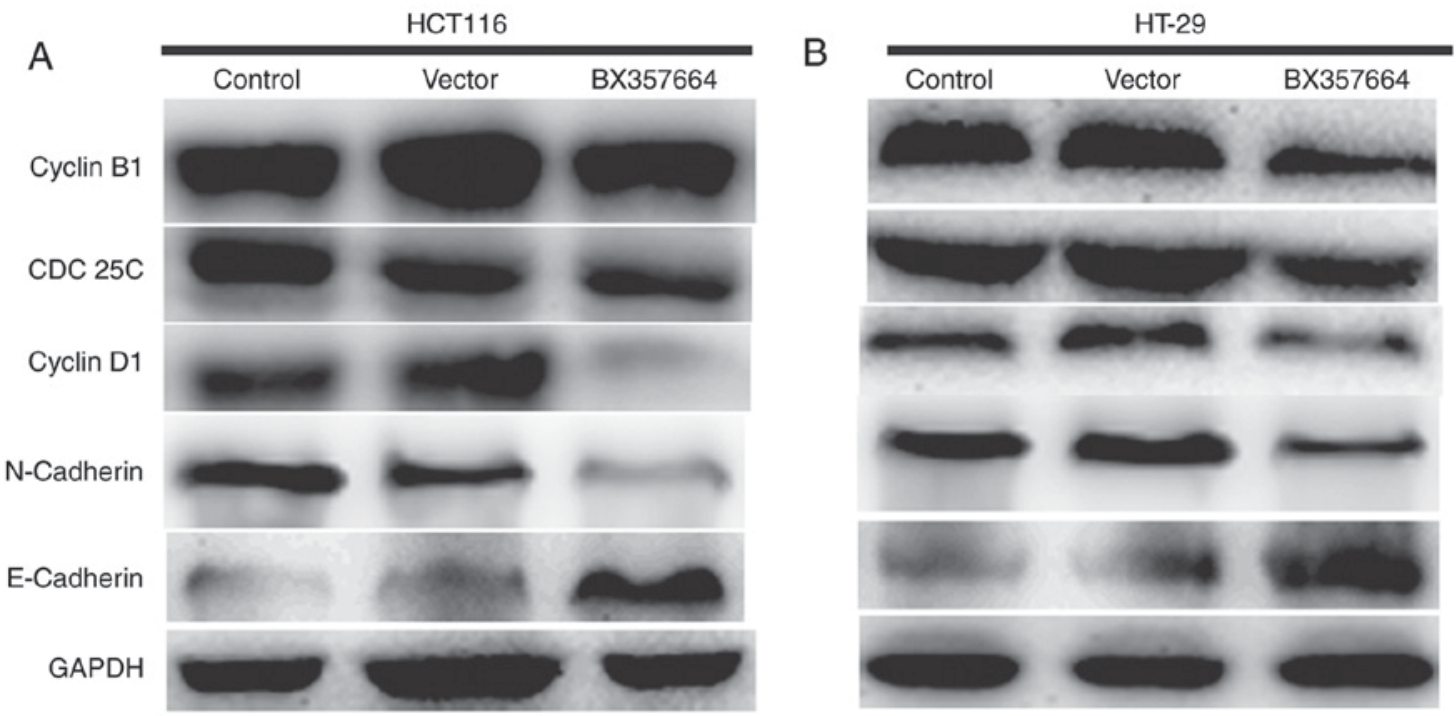

Figure 4. Overexpression of BX357664 alters cell cycle regulator and EMT marker expression. Control non-transfected cells (control), cells transfected with empty vector (vector) or cells transfected with BX357664-expressing plasmid were analyzed by western blotting for protein levels of Cyclin B1, CDC25C, Cyclin D1 and EMT markers E-cadherin and N-cadherin. Representative images of blot results for (A) HCT116 and (B) HT-29 cells are shown. GAPDH was used as a loading control. EMT, epithelial-to-mesenchymal transition; CDC, cell-division cycle.
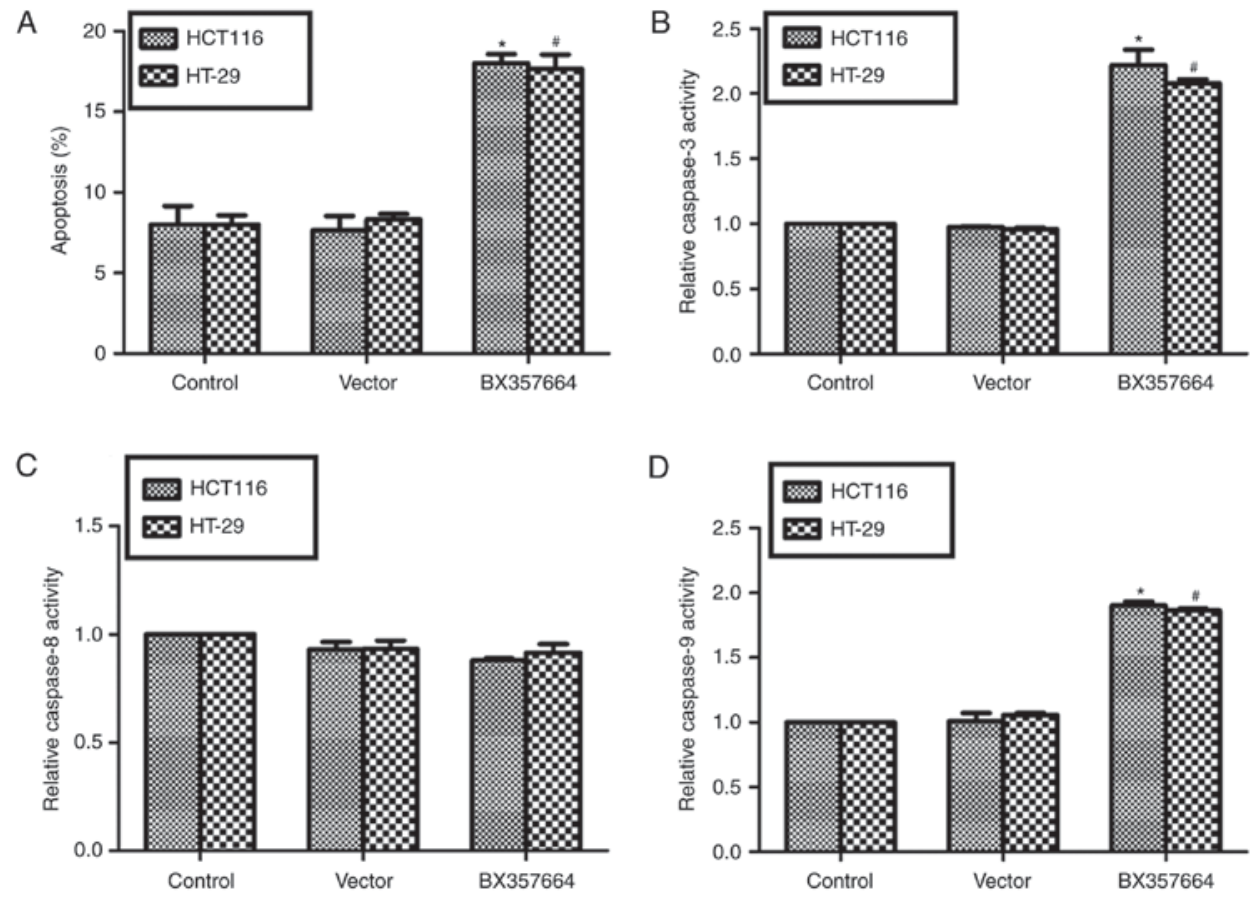

Figure 5. Overexpression of BX357664 promotes cell apoptosis in HCT116 and HT-29 cells. (A) Cell apoptosis was detected in control, vector or BX357664-transfected cells. The cell apoptosis rate was calculated and plotted for each group. The activities of (B) caspase-3, (C) caspase-8 and (D) caspase-9 were determined in control, vector and BX357664-overexpressing HCT116 and HT-29 cells. ${ }^{\text {P }}<0.05$ vs. HCT116 vector group; ${ }^{\# P}<0.05$ vs. HT 29 vector group.

of the epithelial marker E-cadherin was upregulated, while expression of the mesenchymal marker N-cadherin was inhibited following overexpression of BX357664 (Fig. 4A). Similar results were also observed in HT-29 cells (Fig. 4B). These data suggested that overexpression of BX357664 altered the cell cycle progression and EMT processes.

Overexpression of BX357664 promotes cell apoptosis in HCT116 and HT-29 cells. Since BX357664 overexpression arrested cell cycle at G0/G1 phase, we further assessed whether cell apoptosis was regulated by BX357664 overexpression. The results demonstrated that the cell apoptosis rate was $\sim 2$-fold increased following transfection of BX357664 into HCT116 cells or HT-29 cells, compared with the control groups (Fig. 5A). Detection of key apoptosis executors revealed that the activity of caspase- 3 was significantly enhanced following BX357664 overexpression in both CRC cell lines (Fig. 5B). The activity of caspase- 8 remained largely unchanged among the treatment groups (Fig. 5C). The activity of caspase-9 was significantly enhanced by overexpression of BX357664 in 
both HCT116 cells and HT-29 cells (Fig. 5D). These findings suggested that cell apoptosis was significantly induced by BX357664 overexpression in CRC cells.

\section{Discussion}

Colorectal cancer (CRC) represents a great burden for patients worldwide. It is reported that $>1.2$ million patients are diagnosed with CRC annually, of which $\sim 600,000$ die from the disease (5). The cornerstone of therapy for CRC is surgery; however surgery is effective predominantly for localized CRC. In patients suffering from metastatic CRC, only 10-25\% is applicable for surgical resection (13). Despite neoadjuvant radiotherapy (for patients with rectal cancer) and adjuvant chemotherapy (for patients with stage III/IV and high-risk stage II colon cancer), only $30-40 \%$ of patients survive 5 years and over (14). Therefore, new diagnostic and prognostic tools and a detailed understanding of colorectal carcinoma development need to be explored as soon as possible (15).

Accumulating evidence has demonstrated that a body of IncRNAs exert its tissue specificity in various types of cancer and serve an important role in human tumorigenesis and cancer progression $(16,17)$. Emerging lncRNAs have been identified to associate with tumor progression or suppression of CRC, including lncRNA UCC (9), lncRNA CHRF (18) and lncRNA TUSC7 (19). BX357664 was initially identified by microarray analysis in renal cell carcinoma, and its full-length is $650 \mathrm{nt}$. BX357664 was predicted to have the least potential of protein coding by the online Coding Potential Assessment Tool (20). BX357664 was reported to be downregulated in renal cell carcinoma and to negatively regulate the cell proliferation and EMT processes (11). However, the role of BX357664 in other solid tumors remains largely unknown. In view that the same molecule may play different roles in different types of cancer, the role of BX357664 in other tumors merits further exploration. This detailed information would largely enhance our understanding of human tumorigenesis and of the functional roles of BX357664 in human cancers.

The present study examined the role of BX357664 in human CRC, and it represents the first report about this IncRNA in human CRC. Similarly to the report demonstrating that BX357664 negatively regulated cell proliferation and EMT in human renal carcinoma (11), the present study also demonstrated a significant role of BX357664 on human CRC. First, the present study examined the downregulation of this IncRNA in human CRC tissues and cultured cells, which was consistent with that in renal carcinoma (11). Second, based on its lower transcript level in CRC, BX357664 was overexpressed in two CRC cell lines, HCT116 and HT-29, and the effects on cell proliferation, migration and invasion were examined. Consistent with the observations in renal carcinoma (11), overexpression of BX357664 led to significantly decreased cell proliferation, migration and invasion capacities in CRC cells.

To further verify the observations in cell proliferation, migration and invasion, additional cell cycle and western blot analysis of cell cycle regulators and EMT markers were performed. Cell cycle arrest is a hallmark of proliferation inhibition and an indicator of tumor suppression (21). Cyclin B1, CDC25C and Cyclin D1 are key regulators of the cell cycle. Deregulation of these proteins is associated with aberrant tumor growth (21). The present western blot analysis revealed that the protein levels of Cyclin B1, CDC25C and Cyclin D1 were consistently decreased following overexpression of BX357664 in CRC cells, consistent with the proliferation inhibition mediated by BX357664. Additionally, EMT, the process where tumor cells obtain highly mesenchymal properties and lose the epithelial phenotype, is significantly associated with metastasis (21). N-cadherin is a mesenchymal marker and E-cadherin is an epithelial marker (22). The present western blot analysis detected that expression of the mesenchymal marker N-cadherin was decreased while the epithelial marker E-cadherin was increased following BX357664 overexpression in CRC cells, consistent with the migration and invasion inhibition effects mediated by BX357664. Therefore, the present results strongly suggested that BX357664 exerted inhibition effects on CRC proliferation, migration and invasion.

Third, cell cycle arrest results in cell apoptosis in human cancers $(23,24)$. Therefore, the role of BX357664 in cell apoptosis was further examined. The present results demonstrated that BX357664 overexpression significantly increased cell apoptosis rates, which further supported the cell cycle arrest and cell proliferation inhibition mediated by BX357664 in CRC cells. Taken together, the present findings were consistent with the previous study in renal cell carcinoma (11), and suggest that BX357664 may serve as a tumor suppressor in a wide range of solid tumors.

The mechanisms by which BX357664 exerts its functions in CRC remain to be elucidated. The present data demonstrated that cell apoptosis was induced following overexpression of BX357664. The activities of caspase-3 and caspase-9, but not caspase- 8 , were significantly enhanced by BX357664 overexpression. These data indicated that BX357664 might inhibit CRC progression via a caspase-3 and caspase-9-dependent apoptosis pathway. Since BX357664 is a novel lncRNA whose role in solid tumors remains largely unknown, and there exists only one previous study that reported that TGF- $\beta 1 / \mathrm{p} 38 / \mathrm{HSP} 27$ signaling was regulated by BX357664 in renal cell carcinoma (11), the molecular mechanisms by which BX357664 exerts its properties in CRC would merit further investigation.

In summary, the present study demonstrated that BX357664 was downregulated in clinical CRC tissues compared with adjacent normal tissues. Overexpression of BX357664 decreased cell proliferation, migration and invasion capacities in CRC cells. The present data suggested that BX357664 might have critical suppressive effects on human tumorigenesis and serve as a tumor suppressor. These data provided evidence that overexpression of BX357664 might be a valuable therapeutic strategy for a wide range of solid tumors, such as CRC and renal cell carcinoma.

\section{References}

1. Ferlay J, Parkin DM and Steliarova-Foucher E: Estimates of cancer incidence and mortality in Europe in 2008. Eur J Cancer 46: 765-781, 2010.

2. Bray F, Ren JS, Masuyer E and Ferlay J: Global estimates of cancer prevalence for 27 sites in the adult population in 2008. Int J Cancer 132: 1133-1145, 2013.

3. Torre LA, Bray F, Siegel RL, Ferlay J, Lortet-Tieulent J and Jemal A: Global cancer statistics, 2012. CA Cancer J Clin 65: 87-108, 2015. 
4. Guo P, Huang ZL, Yu P and Li K: Trends in cancer mortality in China: An update. Ann Oncol 23: 2755-2762, 2012.

5. Brenner H, Kloor M and Pox CP: Colorectal cancer. Lancet 383 . 1490-1502, 2014

6. de Hoon M, Shin JW and Carninci P: Paradigm shifts in genomics through the FANTOM projects. Mamm Genome 26: 391-402, 2015.

7. Martens-Uzunova ES, Bottcher R, Croce CM, Jenster G, Visakorpi T and Calin GA: Long noncoding RNA in prostate, bladder and kidney cancer. Eur Urol 65: 1140-1151, 2014.

8. Chen L, Wang W, Cao L, Li Z and Wang X: Long Non-Coding RNA CCAT1 acts as a competing endogenous RNA to regulate cell growth and differentiation in acute myeloid leukemia. Mol Cells 39: 330-336, 2016.

9. Huang FT, Chen WY, Gu ZQ, Zhuang YY, Li CQ, Wang LY, Peng JF, Zhu Z, Luo X, Li YH, et al: The novel long intergenic noncoding RNA UCC promotes colorectal cancer progression by sponging miR-143. Cell Death Dis 8: e2778, 2017.

10. Qin C, Han Z, Qian J, Bao M, Li P, Ju X, Zhang S, Zhang L, Li S Cao Q, et al: Expression pattern of long non-coding RNAs in renal cell carcinoma revealed by microarray. PLoS One 9: e99372, 2014

11. Liu Y, Qian J, Li X, Chen W, Xu A, Zhao K, Hua Y, Huang Z, Zhang J, Liang C, et al: Long noncoding RNA BX357664 regulates cell proliferation and epithelial-to-mesenchymal transition via inhibition of TGF- $\beta 1 / \mathrm{p} 38 / \mathrm{HSP} 27$ signaling in renal cell carcinoma. Oncotarget 7: 81410-81422, 2016.

12. Livak KJ and Schmittgen TD: Analysis of relative gene expression data using real-time quantitative PCR and the 2(-Delta Delta C(T)) method. Methods 25: 402-408, 2001

13. Tomlinson JS, Jarnagin WR, DeMatteo RP, Fong Y Kornprat $\mathrm{P}$, Gonen M, Kemeny N, Brennan MF, Blumgart LH and D'Angelica M: Actual 10-year survival after resection of colorectal liver metastases defines cure. J Clin Oncol 25: 4575-4580, 2007.

14. Abdalla EK, Vauthey JN, Ellis LM, Ellis V, Pollock R, Broglio KR, Hess K and Curley SA: Recurrence and outcomes following hepatic resection, radiofrequency ablation and combined resection/ablation for colorectal liver metastases. Ann Surg 239: 818-827, 2004.
15. $\mathrm{Li} \mathrm{L}$ and Luo HS: G-Protein signaling protein-17 (RGS17) is upregulated and promotes tumor growth and migration in human colorectal carcinoma. Oncol Res, 2017 (Epub ahead of print).

16. Prensner JR, Iyer MK, Balbin OA, Dhanasekaran SM, Cao Q, Brenner JC, Laxman B, Asangani IA, Grasso CS, Kominsky HD, et al: Transcriptome sequencing across a prostate cancer cohort identifies PCAT-1, an unannotated lincRNA implicated in disease progression. Nat Biotechnol. 29: 742-749, 2011.

17. Li H, Ma SQ, Huang J, Chen XP and Zhou HH: Roles of long noncoding RNAs in colorectal cancer metastasis. Oncotarget 8: 39859-39876, 2017

18. Tao Y,Han T, Zhang T, Ma C and Sun C: LncRNA CHRF-induced miR-489 loss promotes metastasis of colorectal cancer via TWIST1/EMT signaling pathway. Oncotarget 30: 36410-36422, 2017.

19. Xu J, Zhang $\mathrm{R}$ and Zhao J: The novel long noncoding RNA TUSC7 inhibits proliferation by sponging MiR-211 in colorectal cancer. Cell Physiol Biochem 41: 635-644, 2017.

20. Wang L, Park HJ, Dasari S, Wang S, Kocher JP and Li W: CPAT: Coding-Potential assessment tool using an alignment-free logistic regression model. Nucleic Acids Res. 41: e74, 2013.

21. Sherr CJ: Cancer cell cycles. Science 274: 1672-1677, 1996.

22. Nieto MA, Huang RY, Jackson RA and Thiery JP: Emt: 2016. Cell 166: 21-45, 2016.

23. Xu XY, Xia P, Yu M, Nie XC, Yang X, Xing YN, Liu YP Takano $Y$ and Zheng HC: The roles of REIC gene and its encoding product in gastric carcinoma. Cell Cycle 11: 1414-1431, 2012

24. Du Y, Gong J, Tian X, Yan X, Guo T, Huang M, Zhang B, $\mathrm{Hu} \mathrm{X}$, Liu H, Wang Y, et al: Japonicone a inhibits the growth of non-small cell lung cancer cells via mitochondria-mediated pathways. Tumour Biol 36: 7473-7482, 2015.

c) (i) $($ This work is licensed under a Creative Commons

C) ${ }_{\mathrm{EY}}$ NO NO Attribution-NonCommercial-NoDerivatives 4.0 International (CC BY-NC-ND 4.0) License. 